\title{
Chapter 20 \\ Sensitivity Analyses of Initial Compositions and Cross Sections for Activation Products of In-Core Structure Materials
}

\author{
Kento Yamamoto, Keisuke Okumura, Kensuke Kojima, \\ and Tsutomu Okamoto
}

\begin{abstract}
Sensitivity analyses of initial compositions and cross sections were conducted to quantitatively clarify the source elements and the nuclear reactions dominating the generation of activation products. In these analyses, the ORIGEN2.2 code was used with ORLIBJ40, a set of the cross-section libraries based on JENDL-4.0. Analyses were conducted for the activations of cladding tubes, end plugs, and spacers of fuel assemblies and channel boxes in BWR that are composed of zirconium alloy, stainless steel, and nickel-chromium-based alloy. From about 50 representative radioactive nuclides, several nuclides were selected as the targets of sensitivity analyses for the aspect of their large concentrations in the target materials.

The results of sensitivity coefficients clarified the source elements and the nuclear reactions dominating the generation of activation products even for the nuclides generated through complicated pathways. These results could be utilized to select the objectives of the impurity elements for measurements and of nuclear data for the improvement of accuracy. These results will contribute to improvements in the accuracy of numerical evaluations of activation product concentrations.
\end{abstract}

Keywords Activation products - Burn-up calculation - INCONEL alloy • ORIGEN2.2 • ORLIBJ40 • Sensitivity study • Stainless steel • Zircaloy

\subsection{Introduction}

In the research on the back-end of nuclear cycles, improvement of the accuracy of predicting concentrations of activation products is important for various evaluations. Providing the accurate initial compositions and nuclear data leading to the generation of activation products is necessary for accurate predictions of

K. Yamamoto $(\bowtie) \cdot K$. Okumura $\bullet$ K. Kojima $・$ T. Okamoto

Japan Atomic Energy Agency, Ibaraki, Japan

e-mail: yamamoto.kento@jaea.go.jp 
concentrations of the activation products. An effective first step to achieving this is to identify the dominant generation pathways of activation products. Sensitivity analyses of initial compositions and cross sections for activation products, which involve understanding the effects of initial compositions and cross sections on the concentrations of the target activation products, are powerful methods for quantitatively investigating the generation pathways. Thus, in the present study, sensitivity analyses focusing on the generation pathways for activation products were conducted.

The ORIGEN2.2 [1] code was used in the analyses; this code has been widely used for evaluating the concentrations of activation products. The one-group cross sections made with the appropriate neutron spectrum are required for the accuracy of the ORIGEN2.2 calculation. With respect to the activations of in-core structure materials, the existing ORIGEN2.2 cross-section libraries made with the in-core neutron spectrum are available. Thus, the target of the analyses in the present study is the activation of such in-core structure materials.

This chapter presents the method, calculation conditions, and results of the sensitivity analyses of initial compositions and cross sections for activation products in the materials of in-core structures, such as zirconium alloy, stainless steel, and nickel-chromium-based alloy. The results of the sensitivity analyses identify the elements and the nuclear reactions leading to the generation of activation products. These results will be effective in improving the accuracy of numerical evaluations of the concentrations of activation products.

\subsection{Method of Calculating Sensitivity Coefficients}

A sensitivity coefficient is defined as the ratio of the variation in concentration of the target activation product to the variation in the initial composition or cross section. The sensitivity coefficient of the initial composition and cross section is expressed by the following equations, respectively:

$$
\begin{aligned}
& S=\frac{\Delta W / W_{0}}{\Delta X / X_{0}} \\
& S=\frac{\Delta W / W_{0}}{\Delta \sigma / \sigma_{0}}
\end{aligned}
$$

$W_{0}$ : Concentration of the target activation product under normal condition $\Delta W\left(=W^{\prime}-W_{0}\right)$ : Variation in concentration of the target activation product $X_{0}$ : Initial concentration of the element in the material under normal condition $\Delta X\left(=X^{\prime}-X_{0}\right)$ : Variation in initial concentration of the element in the material $\sigma_{0}$ : Cross section under normal condition $\Delta \sigma\left(=\sigma^{\prime}-\sigma_{0}\right)$ : Variation in cross section 
For the calculation of concentration of activation products, ORIGEN2.2 was used with ORLIBJ40 [2], which is a set of the one-group cross-section libraries based on JENDL-4.0 [3]. The sensitivity coefficients are evaluated by executing two different burn-up calculations under normal condition and under compositionchanged or cross-section-changed condition. In the former, the ORIGEN2.2 input files are changed; in the latter, the cross-section library files are changed. Utility programs to evaluate the sensitivity coefficients were prepared and used in these analyses.

\subsection{Sensitivity Analyses}

\subsubsection{Analyses Conditions}

As stated in Sect. 20.1, activations of in-core structure materials, such as cladding tubes, end plugs, and spacers of fuel assemblies and channel boxes, were investigated in this study. The materials of the in-core structures of PWR and BWR are shown in Table 20.1. The compositions of Zircaloy-2, Zircaloy-4, SUS304 stainless steel, and INCONEL alloy 718 are shown in Table 20.2. In Table 20.2, the average value of the upper and lower limits of the standard specification was applied to the calculation condition for additive elements and the upper limit was applied for impurity elements. The effect of impurity elements that are not specified in the standard are investigated in Sect. 20.3.4.

Typical conditions of BWR were assumed for the cross-section libraries and the irradiation condition, because the difference between the conditions of PWR and BWR is not so significant for the purpose of this study, which is clarifying the dominant generation pathways of activation products.

The cross-section libraries used in these analyses (Table 20.3) were chosen to correspond to the condition of the void ratio in the axial direction. A library made with an average void ratio (40\%) was applied to cladding tubes, spacers, and channel boxes for which the void ratio varies from $0 \%$ to $70 \%$.

A BWR typical irradiation history consists of four cycles of irradiation of about 377 days with constant flux and 90 days of cooling time in the intervals of irradiation (Fig. 20.1). Considering the period for processing of radioactive wastes,

Table 20.1 Materials of in-core structure

\begin{tabular}{l|l|l}
\hline & BWR & PWR \\
\hline Cladding tube & Zircaloy-2 & Zircaloy-4 \\
\hline Top end plug & SUS304 & $\leftarrow$ \\
\hline Bottom end plug & SUS304 & $\leftarrow$ \\
\hline Spacer & Plate: Zircaloy-2 & Zircaloy-4 or \\
\cline { 2 - 3 } & Spring: INCONEL alloy 718 & INCONEL alloy 718 \\
\hline Channel box & Zircaloy-4 & - \\
\hline
\end{tabular}


Table 20.2 Compositions of materials

\begin{tabular}{l|l|l|l|l}
\hline \multicolumn{4}{l}{ Specification (wt\%) } & \multicolumn{2}{l}{ Value in analysis (wt\%) } \\
\hline (a) Zircaloy-2 (JIS H 4751) & \multicolumn{2}{l}{} \\
\hline $\mathrm{H}$ & 0.0025 & Max. & 0.0025 \\
\hline $\mathrm{B}$ & 0.00005 & Max. & & 0.00005 \\
\hline $\mathrm{C}$ & 0.027 & Max. & & 0.027 \\
\hline $\mathrm{N}$ & 0.008 & Max. & 0.008 \\
\hline $\mathrm{Mg}$ & 0.002 & Max. & & 0.002 \\
\hline $\mathrm{Al}$ & 0.0075 & Max. & & 0.0075 \\
\hline $\mathrm{Si}$ & 0.012 & Max. & & 0.012 \\
\hline $\mathrm{Ca}$ & 0.003 & Max. & & 0.003 \\
\hline $\mathrm{Ti}$ & 0.005 & Max. & 0.005 \\
\hline $\mathrm{Cr}$ & 0.05 & - & 0.15 & 0.10 \\
\hline $\mathrm{Mn}$ & 0.005 & Max. & & 0.005 \\
\hline $\mathrm{Fe}$ & 0.07 & - & 0.20 & 0.135 \\
\hline $\mathrm{Co}$ & 0.002 & Max. & & 0.002 \\
\hline $\mathrm{Ni}$ & 0.03 & - & 0.08 & 0.055 \\
\hline $\mathrm{Cu}$ & 0.005 & Max. & & 0.005 \\
\hline $\mathrm{Zr}$ & & Balance & & 98.1456 \\
\hline $\mathrm{Nb}$ & 0.01 & Max. & & 0.01 \\
\hline $\mathrm{Mo}$ & 0.005 & Max. & & 0.005 \\
\hline $\mathrm{Cd}$ & 0.00005 & Max. & & 0.00005 \\
\hline $\mathrm{Sn}$ & 1.20 & - & 1.70 & 1.45 \\
\hline $\mathrm{Hf}$ & 0.01 & Max. & & 0.01 \\
\hline $\mathrm{W}$ & 0.01 & Max. & & 0.01 \\
\hline $\mathrm{U}$ & 0.00035 & Max. & & 0.00035 \\
\hline $\mathrm{b}) \mathrm{Zirat}$ & & &
\end{tabular}

(b) Zircaloy-4 (JIS H 4751)

\begin{tabular}{l|l|l|l|l}
\hline $\mathrm{H}$ & 0.0025 & Max. & & 0.0025 \\
\hline $\mathrm{B}$ & 0.00005 & Max. & & 0.00005 \\
\hline $\mathrm{C}$ & 0.027 & Max. & & 0.027 \\
\hline $\mathrm{N}$ & 0.008 & Max. & & 0.008 \\
\hline $\mathrm{Mg}$ & 0.002 & Max. & & 0.002 \\
\hline $\mathrm{Al}$ & 0.0075 & Max. & & 0.0075 \\
\hline $\mathrm{Si}$ & 0.012 & Max. & & 0.012 \\
\hline $\mathrm{Ca}$ & 0.003 & Max. & & 0.003 \\
\hline $\mathrm{Ti}$ & 0.005 & Max. & & 0.005 \\
\hline $\mathrm{Cr}$ & 0.07 & - & 0.13 & 0.10 \\
\hline $\mathrm{Mn}$ & 0.005 & Max. & & 0.005 \\
\hline $\mathrm{Fe}$ & 0.18 & - & 0.24 & 0.21 \\
\hline $\mathrm{Co}$ & 0.002 & Max. & & 0.002 \\
\hline $\mathrm{Ni}$ & 0.007 & Max. & & 0.007 \\
\hline $\mathrm{Cu}$ & 0.005 & Max. & & 0.005 \\
\hline $\mathrm{Zr}$ & & Balance & & 98.1186 \\
\hline $\mathrm{Nb}$ & 0.01 & Max. & & 0.01 \\
\hline
\end{tabular}


Table 20.2 (continued)

\begin{tabular}{l|l|l|l|l}
\hline & \multicolumn{2}{|l|}{ Specification (wt\%) } & Value in analysis (wt\%) \\
\hline Mo & 0.005 & Max. & & 0.005 \\
\hline Cd & 0.00005 & Max. & & 0.00005 \\
\hline Sn & 1.20 & - & 1.70 & 1.45 \\
\hline Hf & 0.01 & Max. & & 0.01 \\
\hline W & 0.01 & Max. & & 0.01 \\
\hline U & 0.00035 & Max. & 0.00035 \\
\hline
\end{tabular}

(c) SUS304 stainless steel (JIS G 4303)

\begin{tabular}{l|l|l|l|l}
\hline $\mathrm{C}$ & 0.08 & Max. & & 0.08 \\
\hline $\mathrm{Si}$ & 1.00 & Max. & & 1.00 \\
\hline $\mathrm{P}$ & 0.045 & Max. & & 0.05 \\
\hline $\mathrm{S}$ & 0.030 & Max. & & 0.03 \\
\hline $\mathrm{Cr}$ & 18.00 & - & 20.00 & 19.00 \\
\hline $\mathrm{Mn}$ & 2.00 & Max. & & 2.00 \\
\hline $\mathrm{Fe}$ & & Balance & & 68.595 \\
\hline $\mathrm{Ni}$ & 8.00 & - & 10.50 & 9.25
\end{tabular}

(d) INCONEL alloy 718 (UNS N07718)

\begin{tabular}{l|l|l|l|l}
\hline $\mathrm{B}$ & 0.006 & Max. & & 0.006 \\
\hline $\mathrm{C}$ & 0.08 & Max. & & 0.08 \\
\hline $\mathrm{Al}$ & 0.20 & - & 0.80 & 0.50 \\
\hline $\mathrm{Si}$ & 0.35 & Max. & & 0.35 \\
\hline $\mathrm{P}$ & 0.015 & Max. & & 0.015 \\
\hline $\mathrm{Si}$ & 0.015 & Max. & & 0.015 \\
\hline $\mathrm{Cr}$ & 0.65 & - & 1.15 & 0.90 \\
\hline $\mathrm{Mn}$ & 17.00 & - & 21.00 & 19.00 \\
\hline $\mathrm{Fe}$ & 0.35 & Max. & & 0.35 \\
\hline $\mathrm{Co}$ & & Balance & & 16.809 \\
\hline $\mathrm{Ni}$ & 1.00 & Max. & & 1.00 \\
\hline $\mathrm{Cu}$ & 50.00 & - & 55.00 & 52.50 \\
\hline $\mathrm{Nb}$ & 0.3 & Max. & & 0.30 \\
\hline $\mathrm{Mo}$ & 4.75 & - & 5.50 & 5.125 \\
\hline
\end{tabular}

Table 20.3 Cross-section libraries

\begin{tabular}{l|l}
\hline & Specification in cross-section library \\
\hline Cladding tubes, spacers, channel boxes & BWR STEP-III, void ratio 40\% \\
\hline Top-end-plugs & BWR STEP-III, void ratio 70\% \\
\hline Bottom-end-plugs & BWR STEP-III, void ratio 0 \% \\
\hline
\end{tabular}

10 years of cooling time after irradiation was assumed in these analyses. The flux intensities at the center, top, and bottom in the axial direction are shown in Table 20.4. The flux intensity at the center corresponds to the average power in typical BWR fuel assemblies. The flux intensities at the top and bottom were 


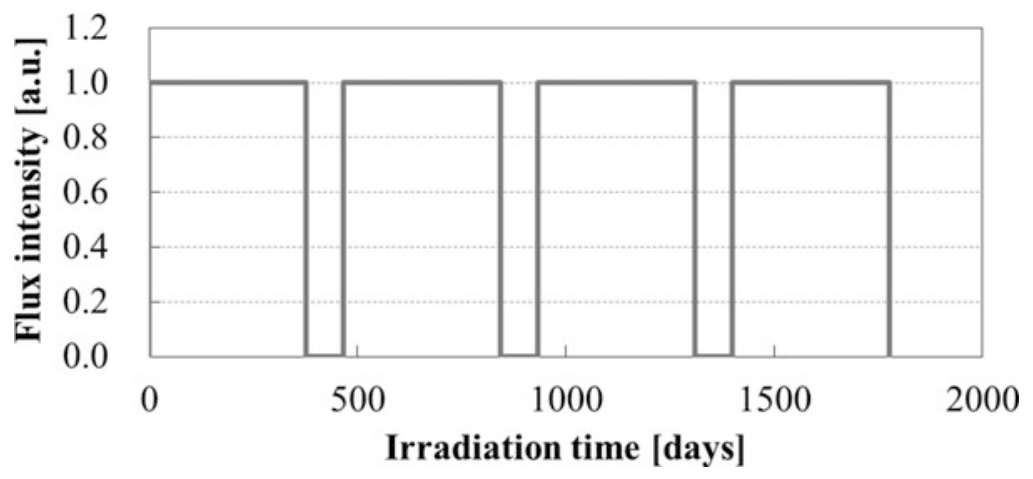

Fig. 20.1 Irradiation history

Table 20.4 Flux intensity at center, top, and bottom in axial direction

\begin{tabular}{l|l}
\hline & Flux intensity $\left(1 / \mathrm{cm}^{2} \mathrm{~s}\right)$ \\
\hline Center & $1.994 \mathrm{E}+14$ \\
\hline Top and bottom & $9.970 \mathrm{E}+12$ \\
\hline
\end{tabular}

determined to be $5 \%$ of that at the center, based on flux distribution evaluated by the one-dimensional neutron diffusion calculation.

\subsubsection{Target Nuclides of Sensitivity Analyses}

The representative radioactive nuclides in this study (Table 20.5) include not only the important nuclides for various evaluations of radioactive wastes but also the nuclides whose concentrations have been measured in the past, which will be useful for the validation of numerical evaluations.

Target nuclides of sensitivity analyses were selected on the basis of two criteria. The first was that the concentrations of activation products be larger than or comparable to the concentration of fission products generated from impurity uranium in the materials. The contents of impurity uranium in Zircaloy-2 and SUS304 stainless steel were $0.00035 \mathrm{wt} \%$ and $0.0001 \mathrm{wt} \%$ [5], respectively. This value in INCONEL alloy is unknown. The second criterion was that the concentrations of activation products be comparatively large. In these analyses, activation products with concentrations more than $1 \times 10^{-9} \mathrm{~g} / \mathrm{t}$ were chosen.

The concentrations of activation products larger than $1 \times 10^{-9} \mathrm{~g} / \mathrm{t}$ and fission products generated from impurity uranium are shown in Table 20.6. The fission products were calculated under the condition that the initial composition contains only the uranium impurity. Table 20.6 also shows the selected target nuclides that satisfy the foregoing criterion: 17 nuclides in Zircaloy-2 and Zircaloy-4, 8 nuclides in SUS304 stainless steel, and 16 nuclides in INCONEL alloy were selected as the target nuclides of sensitivity analyses. 


\begin{tabular}{|c|c|c|c|c|c|c|c|c|c|c|c|c|c|c|c|c|c|}
\hline 5 & II & 胥 & 节 & 齐 & $\sum_{\bar{z}}^{\infty}$ & 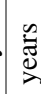 & 离 & $\underset{\tilde{J}}{\stackrel{\Xi}{\Delta}}$ & 量 & 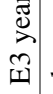 & 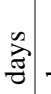 & 荨 & & & & & \\
\hline$?$ & $\begin{array}{l}0 \\
\dot{\infty} \\
\stackrel{0}{0}\end{array}$ & $\stackrel{\overbrace{}}{8}$ & i & $\tilde{n}$ & 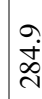 & $\stackrel{n}{2}$ & $\begin{array}{l}0 \\
\infty \\
\infty\end{array}$ & $\stackrel{\infty}{+}$ & 守 & $\stackrel{\sim}{\longrightarrow}$ & $\underset{\sim}{\stackrel{\sim}{\sim}}$ & $\vec{m}$ & & & & & \\
\hline & $\begin{array}{l}\frac{2}{\mathcal{H}} \\
\frac{1}{n}\end{array}$ & $\begin{array}{l}\stackrel{ \pm}{さ} \\
\frac{1}{2} \\
\text { की }\end{array}$ & $\begin{array}{l}a \\
\frac{1}{3} \\
\frac{1}{n}\end{array}$ & $\begin{array}{l}\bar{y} \\
\bar{d} \\
\dot{d}\end{array}$ & $\frac{8}{\dot{J}}$ & $\frac{\tilde{N}}{\frac{\pi}{3}}$ & 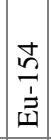 & $\frac{n}{n}$ & $\frac{n}{n}$ & 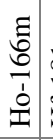 & $\begin{array}{c}\infty \\
\frac{1}{1}\end{array}$ & 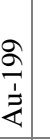 & & & & & \\
\hline $\begin{array}{l}y \\
\text { y }\end{array}$ & $\stackrel{m}{\forall}$ & 寸 & 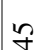 & fo & F & $\stackrel{\infty}{+}$ & g & ก & $\bar{n}$ & กี & $\tilde{n}$ & in & & & & & \\
\hline 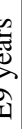 & 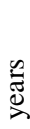 & 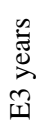 & శ్ & 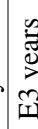 & 胥 & 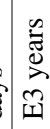 & 号 & 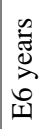 & 挛 & $\stackrel{\infty}{\vec{t}}$ & 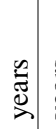 & 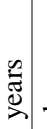 & $\overbrace{\vec{\partial}}^{\infty}$ & 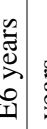 & 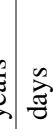 & 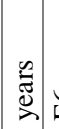 & 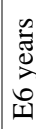 \\
\hline$\infty$ & & $\stackrel{?}{\stackrel{\sim}{\Omega}}$ & के. & $\stackrel{\circ}{+}$ & $\tilde{i}$ & $\exists$ & $\ddot{0}$ & $\because$ & $\stackrel{0}{\stackrel{+}{0}}$ & m. & $\stackrel{0}{-}$ & \begin{tabular}{c|c}
0 & \\
$\infty$ & \\
$\dot{\sigma}$ & \\
$\dot{\sigma}$ &
\end{tabular} & & $\vec{c}$ & $\stackrel{0}{\infty}$ & $\vec{i}$ & $\hat{n}$ \\
\hline
\end{tabular}

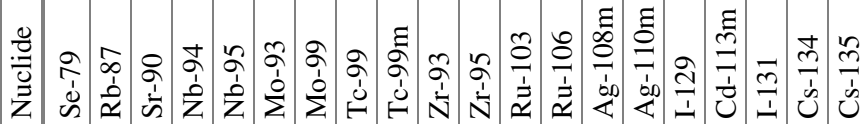

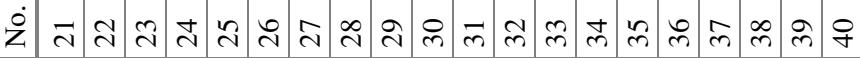

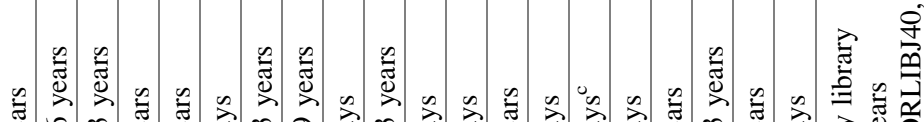

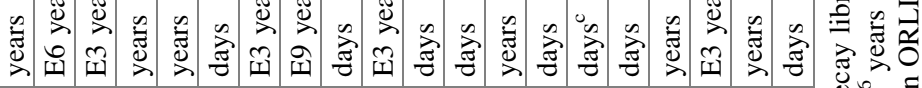

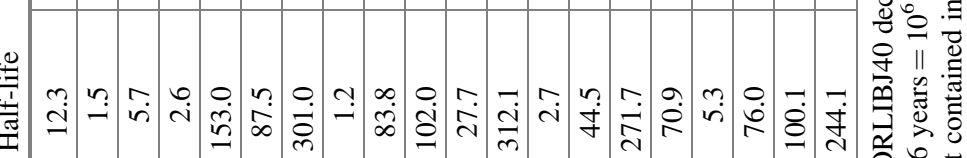
\begin{tabular}{l|l|l|l|l|l|l|l|l|l|l|l|l|l|l} 
& & & & & & & \\
0
\end{tabular}

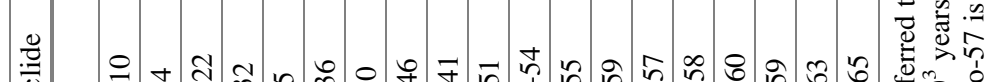

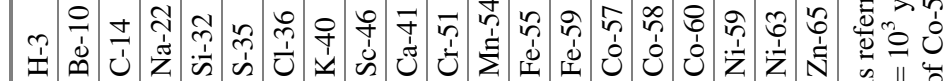

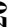

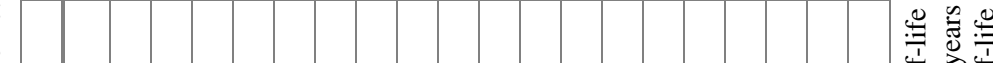

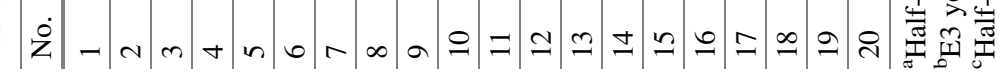


Table 20.6 Concentration of activation products and fission products

\begin{tabular}{|c|c|c|c|c|c|}
\hline \multirow[b]{2}{*}{ Nuclide } & \multicolumn{2}{|c|}{$\begin{array}{l}\text { Concentration of } \\
\text { activation products } \\
(\mathrm{g} / \mathrm{t})\end{array}$} & \multirow{2}{*}{$\begin{array}{l}\text { Concentration of fission } \\
\text { products }(\mathrm{g} / \mathrm{t})\end{array}$} & \multirow{2}{*}{$\begin{array}{l}\text { Comparison } \\
(\%)\end{array}$} & \multirow{2}{*}{$\begin{array}{l}\text { Target } \\
\text { nuclide }\end{array}$} \\
\hline & (1)Zry-2 & (2)Zry-4 & & & \\
\hline
\end{tabular}

(a) Zircaloy-2 and Zircaloy-4

\begin{tabular}{|c|c|c|c|c|c|}
\hline Zr-93 & $2.0 \mathrm{E}+02$ & $2.0 \mathrm{E}+02$ & 1.2E-03 & 0 & 0 \\
\hline Ni-59 & $3.7 \mathrm{E}+00$ & 4.7E-01 & - & - & 0 \\
\hline $\mathrm{Ni}-63$ & 6.6E-01 & $8.9 \mathrm{E}-02$ & - & - & 0 \\
\hline Co-60 & 5.1E-01 & $5.1 \mathrm{E}-01$ & - & - & 0 \\
\hline $\mathrm{C}-14$ & 4.0E-01 & $4.0 \mathrm{E}-01$ & - & - & 0 \\
\hline $\mathrm{Nb}-94$ & 3.0E-01 & $3.0 \mathrm{E}-01$ & 3.2E-09 & 0 & 0 \\
\hline Sb-125 & $2.5 \mathrm{E}-01$ & $2.5 \mathrm{E}-01$ & $1.5 \mathrm{E}-06$ & 0 & 0 \\
\hline $\mathrm{Ca}-41$ & 3.0E-02 & $3.0 \mathrm{E}-02$ & - & - & 0 \\
\hline $\mathrm{K}-40$ & 2.2E-02 & $2.2 \mathrm{E}-02$ & - & - & 0 \\
\hline $\mathrm{Fe}-55$ & 2.1E-02 & $3.2 \mathrm{E}-02$ & - & - & 0 \\
\hline Tc-99 & 9.1E-03 & $9.1 \mathrm{E}-03$ & 1.7E-03 & 16 & 0 \\
\hline Mo-93 & 8.9E-03 & $8.9 \mathrm{E}-03$ & 3.5E-14 & 0 & 0 \\
\hline Be-10 & $4.0 \mathrm{E}-05$ & $4.0 \mathrm{E}-05$ & 2.2E-08 & 0 & 0 \\
\hline Sr-90 & 2.3E-05 & $2.3 \mathrm{E}-05$ & 6.0E-04 & 96 & - \\
\hline Mn-54 & 3.9E-06 & $6.1 \mathrm{E}-06$ & - & - & 0 \\
\hline $\begin{array}{l}\mathrm{Ag}- \\
108 \mathrm{~m}\end{array}$ & 3.3E-07 & $3.3 \mathrm{E}-07$ & 5.6E-12 & 0 & 0 \\
\hline Rb-87 & 1.1E-07 & $1.1 \mathrm{E}-07$ & 3.7E-04 & 100 & - \\
\hline $\mathrm{H}-3$ & 3.0E-08 & $3.0 \mathrm{E}-08$ & 5.8E-08 & 66 & 0 \\
\hline I-129 & 6.4E-09 & 6.4E-09 & 3.9E-04 & 100 & - \\
\hline Zn-65 & 2.8E-09 & $2.8 \mathrm{E}-09$ & - & - & 0 \\
\hline
\end{tabular}

(b) SUS304 stainless steel

\begin{tabular}{l|l|l|l|l|l}
\hline \multirow{2}{*}{ Nuclide } & \multicolumn{2}{l|l|l}{$\begin{array}{l}\text { Concentration of acti- } \\
\text { vation products }(\mathrm{g} / \mathrm{t})\end{array}$} & $\begin{array}{l}\text { Concentration of fission prod- } \\
\text { ucts }(\mathrm{g} / \mathrm{t})\end{array}$ & $\begin{array}{l}\text { Comparison } \\
(\%)\end{array}$ & $\begin{array}{l}\text { Target } \\
\text { nuclide }\end{array}$ \\
\cline { 2 - 6 } & (1) Bottom & 2 Top & 3 & $(3 /(1)+(3))$ & \\
\hline $\mathrm{Ni}-59$ & $4.8 \mathrm{E}+01$ & $2.6 \mathrm{E}+01$ & - & - & 0 \\
\hline $\mathrm{Ni}-63$ & $7.7 \mathrm{E}+00$ & $4.1 \mathrm{E}+00$ & - & - & 0 \\
\hline $\mathrm{Fe}-55$ & $7.0 \mathrm{E}-01$ & $3.9 \mathrm{E}-01$ & - & - & 0 \\
\hline $\mathrm{Co}-60$ & $5.3 \mathrm{E}-03$ & $5.0 \mathrm{E}-03$ & - & - & 0 \\
\hline $\mathrm{Mn}-54$ & $9.9 \mathrm{E}-05$ & $9.9 \mathrm{E}-05$ & - & - & 0 \\
\hline $\mathrm{Be}-10$ & $5.7 \mathrm{E}-06$ & $5.7 \mathrm{E}-06$ & $2.0 \mathrm{E}-10$ & 0 & 0 \\
\hline $\mathrm{C}-14$ & $3.2 \mathrm{E}-06$ & $2.3 \mathrm{E}-06$ & - & - & 0 \\
\hline $\mathrm{Cl}-36$ & $1.6 \mathrm{E}-06$ & $4.4 \mathrm{E}-07$ & - & - & 0 \\
\hline
\end{tabular}


Table 20.6 (continued)

\begin{tabular}{|c|c|c|c|c|c|}
\hline \multirow[b]{2}{*}{ Nuclide } & \multicolumn{2}{|c|}{$\begin{array}{l}\text { Concentration of } \\
\text { activation products } \\
(\mathrm{g} / \mathrm{t})\end{array}$} & \multirow{2}{*}{$\begin{array}{l}\text { Concentration of fission } \\
\text { products }(\mathrm{g} / \mathrm{t}) \\
\text { (3) }\end{array}$} & \multirow{2}{*}{$\begin{array}{l}\text { Comparison } \\
(\%) \\
(3 /(1)+(3))\end{array}$} & \multirow{2}{*}{$\begin{array}{l}\text { Target } \\
\text { nuclide }\end{array}$} \\
\hline & (1)Zry-2 & (2)Zry-4 & & & \\
\hline \multicolumn{6}{|c|}{ (c) INCONEL alloy 718} \\
\hline Nuclide & \multicolumn{4}{|c|}{ Concentration of activation products $(\mathrm{g} / \mathrm{t})$} & $\begin{array}{l}\text { Target } \\
\text { nuclide }\end{array}$ \\
\hline $\mathrm{Ni}-59$ & \multicolumn{4}{|l|}{$3.5 \mathrm{E}+03$} & O \\
\hline $\mathrm{Ni}-63$ & \multicolumn{4}{|l|}{$6.2 \mathrm{E}+02$} & O \\
\hline Co-60 & \multicolumn{4}{|l|}{$2.6 \mathrm{E}+02$} & O \\
\hline $\mathrm{Nb}-94$ & \multicolumn{4}{|l|}{$1.6 \mathrm{E}+02$} & O \\
\hline Mo-93 & \multicolumn{4}{|l|}{$5.4 \mathrm{E}+00$} & O \\
\hline Tc-99 & \multicolumn{4}{|l|}{$5.4 \mathrm{E}+00$} & O \\
\hline $\mathrm{Fe}-55$ & \multicolumn{4}{|l|}{$3.4 \mathrm{E}+00$} & O \\
\hline Zr-93 & \multicolumn{4}{|l|}{$1.3 \mathrm{E}-01$} & O \\
\hline Mn-54 & \multicolumn{4}{|l|}{$4.8 \mathrm{E}-04$} & 0 \\
\hline $\mathrm{Be}-10$ & \multicolumn{4}{|l|}{$2.8 \mathrm{E}-04$} & O \\
\hline $\mathrm{Cl}-36$ & \multicolumn{4}{|l|}{$1.7 \mathrm{E}-04$} & O \\
\hline $\mathrm{C}-14$ & \multicolumn{4}{|l|}{$5.4 \mathrm{E}-05$} & O \\
\hline $\mathrm{Zn}-65$ & \multicolumn{4}{|l|}{$1.7 \mathrm{E}-07$} & O \\
\hline Sr-90 & \multicolumn{4}{|l|}{$1.3 \mathrm{E}-08$} & O \\
\hline Si-32 & \multicolumn{4}{|l|}{ 7.9E-09 } & O \\
\hline $\mathrm{H}-3$ & \multicolumn{4}{|l|}{$1.8 \mathrm{E}-09$} & O \\
\hline
\end{tabular}

\subsubsection{Results of Sensitivity Analyses}

Sensitivity analyses were conducted for several selected nuclides in Zircaloy-2, SUS304 stainless steel, and INCONEL alloy. Analyses in Zircaloy-4 were skipped because the sensitivity coefficients were thought to be almost the same as that in Zircaloy-2 because calculation conditions were similar. For SUS304 stainless steel, activations using the cross-section library of void ratio $0 \%$ were evaluated because the concentrations in the case of void ratio $0 \%$ were larger than that of void ratio $70 \%$.

The sensitivity coefficients of initial compositions are shown in Table 20.7. As defined in Eq. (20.1), the value shows the relative amount of variation in concentration of the target nuclide when the initial composition of element varies by a unit amount. Therefore, the source elements leading to the generation of target nuclides was clarified from the results. For example, Table 20.7a shows that Fe-55 is generated from both iron and nickel and that the contribution from iron is dominant. The results can also be useful in the evaluation of the error propagated from the measurement uncertainty of initial composition.

As defined in Eq. (20.2), a sensitivity coefficient of a cross section shows the relative amount of variation in the concentration of the target nuclide when the 


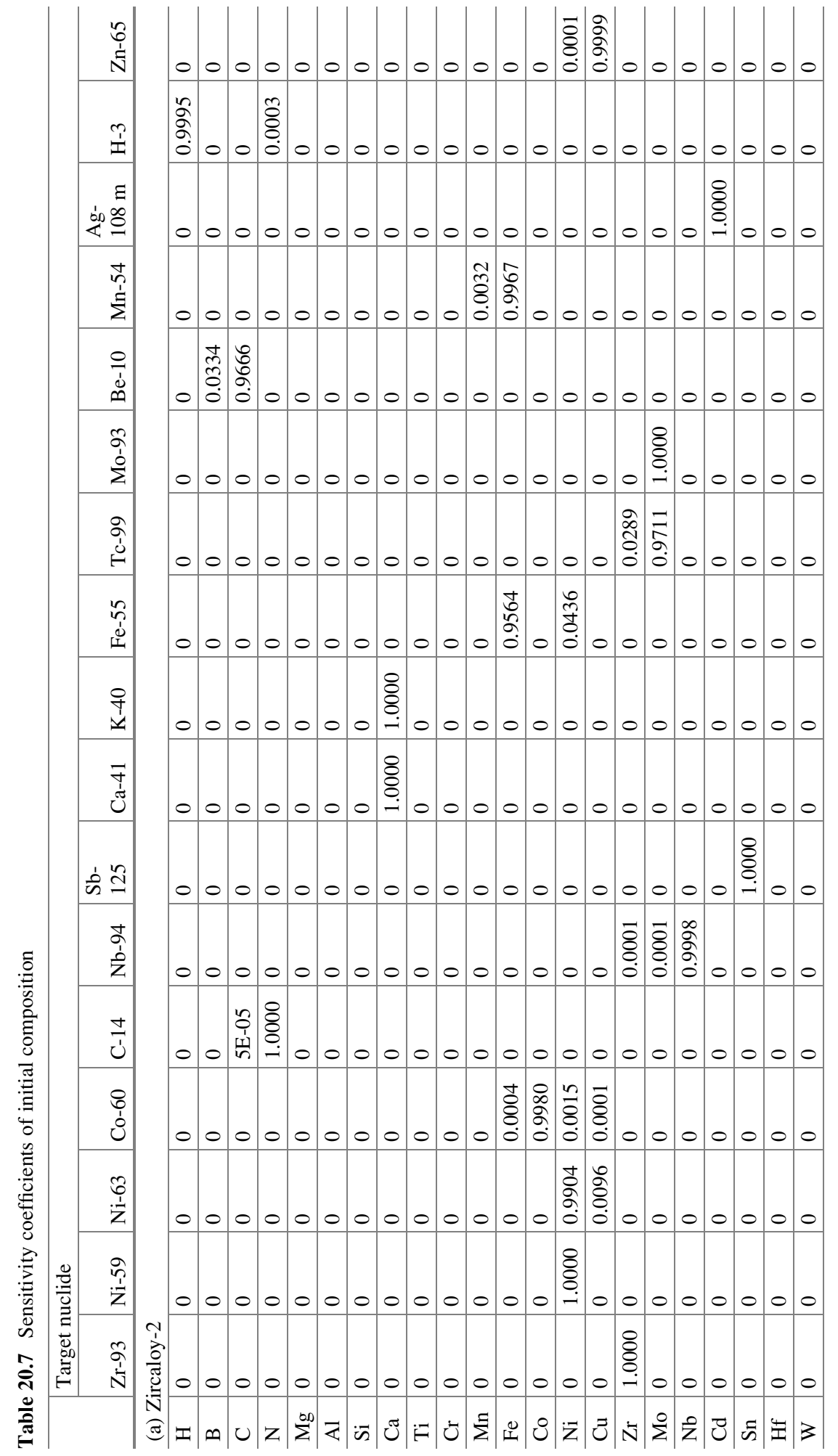




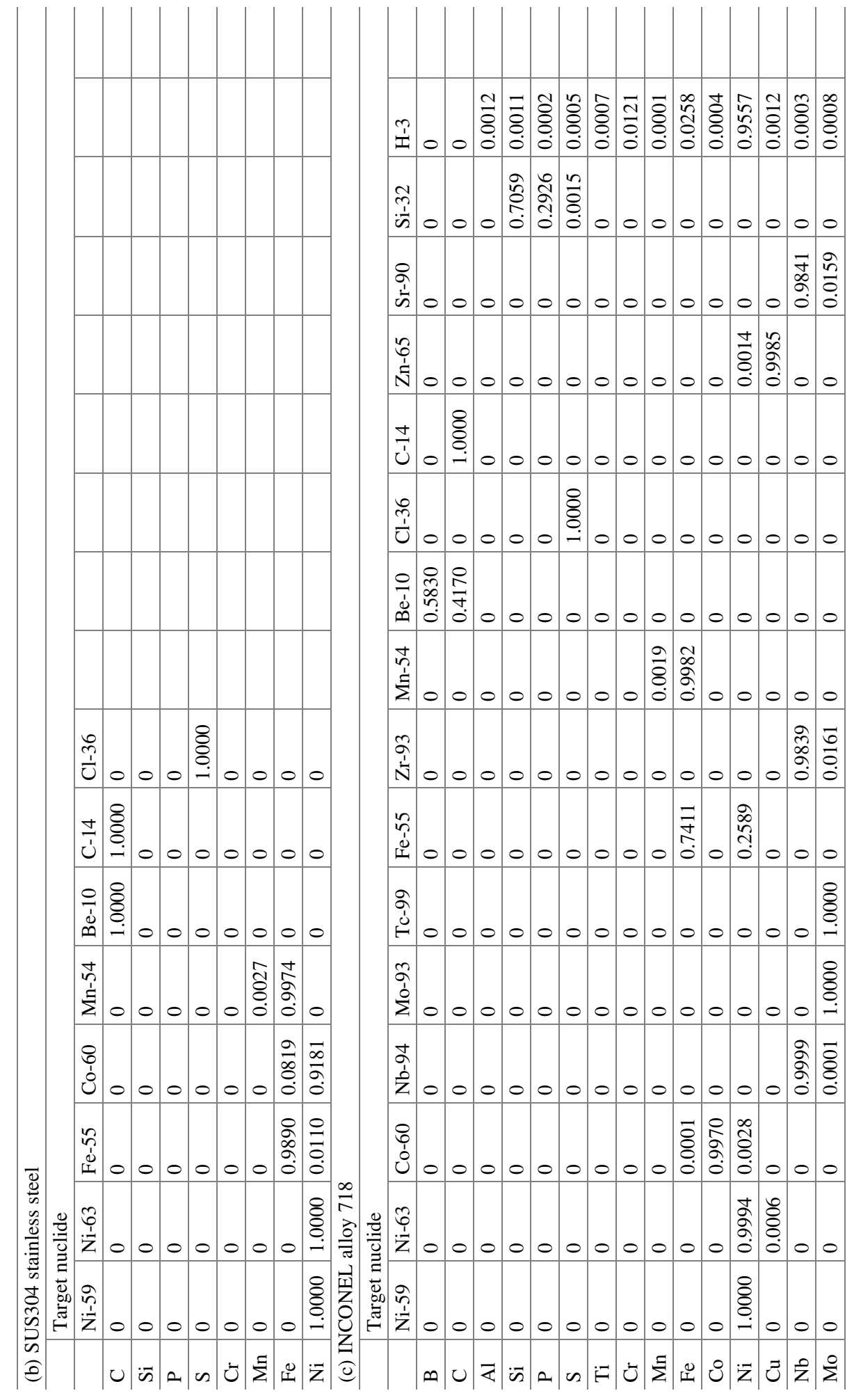


Table 20.8 Sensitivity coefficients of cross sections

\begin{tabular}{|c|c|c|c|c|c|c|c|c|c|}
\hline \multirow[b]{2}{*}{ Target nuclide } & \multicolumn{9}{|c|}{ Sensitivity coefficient of cross section } \\
\hline & \multicolumn{3}{|c|}{ First largest } & \multicolumn{3}{|c|}{ Second largest } & \multicolumn{3}{|l|}{ Others } \\
\hline \multicolumn{10}{|l|}{ (a) Zircaloy-2 } \\
\hline Zr-93 & Zr-92 & $(n, \gamma)$ & 0.98 & Zr-94 & $(n, 2 n)$ & 0.02 & & & \\
\hline $\mathrm{Ni}-59$ & Ni-58 & $(n, \gamma)$ & 0.99 & & & & & & \\
\hline $\mathrm{Ni}-63$ & $\mathrm{Ni}-62$ & $(n, \gamma)$ & 0.97 & & & & & & \\
\hline Co-60 & Co-59 & $(n, \gamma)_{m}$ & 0.46 & Co-59 & $(n, \gamma)$ & 0.42 & & & \\
\hline $\mathrm{C}-14$ & $\mathrm{~N}-14$ & $(n, p)$ & 1.00 & & & & & & \\
\hline $\mathrm{Nb}-94$ & $\mathrm{Nb}-93$ & $(n, \gamma)$ & 1.00 & & & & & & \\
\hline Sb-125 & Sn-124 & $(n, \gamma)$ & 0.56 & Sn-124 & $(n, \gamma)_{m}$ & 0.48 & & & \\
\hline $\mathrm{Ca}-41$ & $\mathrm{Ca}-40$ & $(n, \gamma)$ & 1.00 & & & & & & \\
\hline $\mathrm{K}-40$ & $\mathrm{Ca}-40$ & $(n, p)$ & 1.00 & & & & & & \\
\hline $\mathrm{Fe}-55$ & Fe-54 & $(n, \gamma)$ & 0.95 & $\mathrm{Ni}-58$ & $(n, \alpha)$ & 0.04 & & & \\
\hline Tc-99 & Mo-98 & $(n, \gamma)$ & 1.00 & Mo-97 & $(n, \gamma)$ & 0.03 & $\mathrm{Zr}-96$ & $(n, \gamma)$ & 0.03 \\
\hline Mo-93 & Mo-92 & $(n, \gamma)$ & 0.99 & & & & & & \\
\hline Be-10 & C-13 & $(n, \alpha)$ & 0.97 & B-10 & $(n, p)$ & 0.03 & & & \\
\hline Mn-54 & $\mathrm{Fe}-54$ & $(n, p)$ & 1.00 & & & & & & \\
\hline Ag-108 m & Cd-106 & $(n, \gamma)$ & 1.00 & Ag-107 & $(n, \gamma)_{m}$ & 0.97 & & & \\
\hline $\mathrm{H}-3$ & $\mathrm{H}-2$ & $(n, \gamma)$ & 1.00 & $\mathrm{H}-1$ & $(n, \gamma)$ & 0.78 & $\mathrm{He}-3$ & $(n, p)$ & 0.01 \\
\hline $\mathrm{Zn}-65$ & Zn-64 & $(n, \gamma)$ & 1.00 & $\mathrm{Cu}-63$ & $(n, \gamma)$ & 1.00 & & & \\
\hline
\end{tabular}

(b) SUS304 stainless steel

\begin{tabular}{l|l|l|l|l|l|l|l|l|l}
\hline Ni-59 & Ni-58 & $(n, \gamma)$ & 1.00 & & & & & & \\
\hline Ni-63 & Ni-62 & $(n, \gamma)$ & 1.00 & & & & & & \\
\hline Fe-55 & Fe-54 & $(n, \gamma)$ & 0.99 & Ni-58 & $(n, \alpha)$ & 0.01 & & & \\
\hline Co-60 & Ni-60 & $(n, \mathrm{p})$ & 0.92 & Fe-58 & $(n, \gamma)$ & 0.08 & Co-59 & $(n, \gamma)$ & 0.04 \\
\hline & & & & & & & Co-59 & $(n, \gamma)_{m}$ & 0.04 \\
\hline Mn-54 & Fe-54 & $(n, p)$ & 1.00 & & & & & & \\
\hline Be-10 & C-13 & $(n, \alpha)$ & 1.00 & & & & & & \\
\hline C-14 & C-13 & $(n, \gamma)$ & 1.00 & & & & & & \\
\hline Cl-36 & S-34 & $(n, \gamma)$ & 1.00 & Cl-35 & $(n, \gamma)$ & 1.00 & & & \\
\hline
\end{tabular}

(c) INCONEL alloy 718

\begin{tabular}{l|l|l|l|l|l|l|l|l|l}
\hline Ni-59 & Ni-58 & $(n, \gamma)$ & 0.99 & & & & & & \\
\hline Ni-63 & Ni-62 & $(n, \gamma)$ & 0.98 & & & & & & \\
\hline Co-60 & Co-59 & $(n, \gamma)_{m}$ & 0.46 & Co-59 & $(n, \gamma)$ & 0.42 & & & \\
\hline Nb-94 & Nb-93 & $(n, \gamma)$ & 1.00 & & & & & & \\
\hline Mo-93 & Mo-92 & $(n, \gamma)$ & 0.99 & & & & & & \\
\hline Tc-99 & Mo-98 & $(n, \gamma)$ & 1.00 & & & & & & \\
\hline Fe-55 & Fe-54 & $(n, \gamma)$ & 0.74 & Ni-58 & $(n, \alpha)$ & 0.26 & & & \\
\hline Zr-93 & Nb-93 & $(n, p)$ & 0.98 & Mo-96 & $(n, \alpha)$ & 0.02 & & & \\
\hline Mn-54 & Fe-54 & $(n, p)$ & 1.00 & & & & & & \\
\hline Be-10 & B-10 & $(n, p)$ & 0.58 & C-13 & $(n, \alpha)$ & 0.42 & & & \\
\hline Cl-36 & S-34 & $(n, \gamma)$ & 1.00 & Cl-35 & $(n, \gamma)$ & 0.97 & & & \\
\hline C-14 & C-13 & $(n, \gamma)$ & 1.00 & & & & & & \\
\hline
\end{tabular}


Table 20.8 (continued)

\begin{tabular}{l|l|l|l|l|l|l|l|l|l}
\hline \multirow{2}{*}{ Target nuclide } & \multicolumn{9}{l}{ Sensitivity coefficient of cross section } \\
\cline { 2 - 13 } & First largest & \multicolumn{3}{l|}{ Second largest } & \multicolumn{2}{l}{ Others } \\
\hline Zn-65 & Zn-64 & $(n, \gamma)$ & 0.99 & $\mathrm{Cu}-63$ & $(n, \gamma)$ & 0.99 & & & \\
\hline Sr-90 & Zr-93 & $(n, \alpha)$ & 1.00 & Nb-93 & $(n, p)$ & 0.98 & & & \\
\hline Si-32 & $\mathrm{Si}-31$ & $(n, \gamma)$ & 1.00 & $\mathrm{Si}-30$ & $(n, \gamma)$ & 0.71 & $\mathrm{P}-31$ & $(n, p)$ & 0.29 \\
\hline $\mathrm{H}-3$ & $\mathrm{H}-2$ & $(n, \gamma)$ & 1.00 & $\mathrm{H}-1$ & $(n, \gamma)$ & 1.00 & $\mathrm{Ni}-58$ & $(n, p)$ & 0.95 \\
\hline & & & & & & & $\mathrm{He}-3$ & $(n, p)$ & 0.01 \\
\hline
\end{tabular}

$(n, \gamma)_{m}$ means the $(n, \gamma)$ reaction yielding to meta-stable state

a

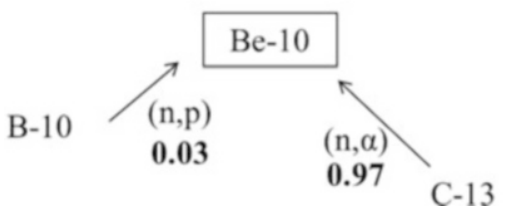

b

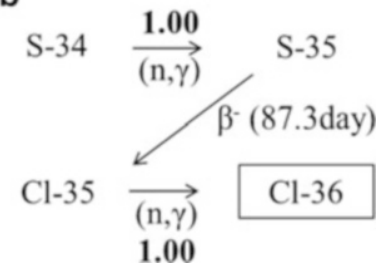

Fig. 20.2 Examples of complicated generation pathways of activation products. The values in bold font in the figures show the sensitivity coefficients of the cross sections. a Be-10 generation in Zircaloy-2. b Cl-36 generation in SUS304 stainless steel

cross section varies by a unit amount. Therefore, a positive value of this coefficient indicates that the target activation product is generated through the nuclear reaction. Thus, if a sensitivity coefficient is positive and large, the cross section of the nuclear reaction is significant for the generation of the target activation products. In the analyses, the objectives of reaction were six reactions treated in ORLIBJ40 library; the reaction of $(n, \gamma),(n, 2 n),(n, \alpha)$, and $(n, p)$ yielding to nuclides of ground state and the reaction of $(n, \gamma)$ and $(n, 2 n)$ yielding to nuclides of meta-stable state. The summary of the results of sensitivity analyses of cross sections are shown in Table 20.8, where the sensitivity coefficients that are positive and more than 0.01 are extracted from all the results and listed in descending order. The results clarified the nuclear reaction dominating the generation of target nuclides. For example, it is thought that Fe-55 in Zircaloy-2 can be generated from the $(n, \gamma)$ reaction of Fe-54, the $(n, \alpha)$ reaction of $\mathrm{Ni}-58$, and the $(n, 2 n)$ reaction of Fe-56. Table 20.8a clearly shows the $(n, \gamma)$ reaction of Fe-54 is dominant in the generation of Fe-55.

It was remarkable that the dominant generation pathways were clarified even for the target nuclides generated through complicated pathways. Some of the examples are shown in Fig. 20.2.

Figure 20.2a shows an example of nuclides generated with some contributing pathways. Be-10 is generated in Zircaloy-2 mainly through two pathways, the $(n, p)$ reaction of $\mathrm{Be}-10$ and the $(n, \alpha)$ reaction of $\mathrm{C}-13$. It is not predictable which pathway is dominant from the initial composition of the material. The sensitivity 
Table 20.9 Initial composition of SUS304 stainless steel

\begin{tabular}{l|l|l}
\hline Element & Value based on measurement data & Value based on the standard specification \\
\hline $\mathrm{C}$ & - & 0.08 \\
\hline $\mathrm{N}$ & 0.05 & - \\
\hline $\mathrm{Si}$ & - & 1.00 \\
\hline $\mathrm{P}$ & - & 0.045 \\
\hline $\mathrm{S}$ & 0.004 & 0.030 \\
\hline $\mathrm{Cl}$ & 0.001 & - \\
\hline $\mathrm{K}$ & $4.0 \mathrm{E}-05$ & - \\
\hline $\mathrm{Cr}$ & - & 19.00 \\
\hline $\mathrm{Mn}$ & - & 2.00 \\
\hline $\mathrm{Fe}$ & 72 & 68.60 \\
\hline $\mathrm{Co}$ & 0.1 & - \\
\hline $\mathrm{Ni}$ & 9.25 & 9.25 \\
\hline $\mathrm{Cu}$ & 0.16 & - \\
\hline $\mathrm{Zr}$ & 0.00032 & - \\
\hline $\mathrm{Nb}$ & 0.02 & - \\
\hline $\mathrm{Mo}$ & 0.13 & - \\
\hline $\mathrm{Th}$ & $2.0 \mathrm{E}-07$ & - \\
\hline $\mathrm{U}$ & $2.0 \mathrm{E}-07$ & 0.0001 \\
\hline
\end{tabular}

coefficients clearly showed that the $(n, \alpha)$ reaction of C-13 is the dominant pathway for Be-10 generation in Zircaloy-2.

Figure $20.2 \mathrm{~b}$ shows an example of nuclides generated through long and complicated generation chains. The source nuclide of Cl-36 generated in SUS304 stainless steel is ambiguous because the initial composition in this analysis does not contain chlorine, which could be the dominant source element of Cl-36. The sensitivity coefficients quantitatively clarified that S-34 is the source nuclide of Cl-36 even for the long and complicated chain.

\subsubsection{Sensitivity Analysis Using the Initial Composition Based on Measured Data}

The sensitivity coefficients shown in Sect. 20.3.3 are valid within the assumed analysis conditions in Sect. 20.3.1. However, the impurity elements that are not specified in the standard specification can be possibly present in the material. To know the effect of the difference in the initial composition on sensitivity coefficients, additional analyses were conducted using the initial composition based on measured data. The evaluation of activation products in SUS304 stainless steel is described here. 
Table 20.10 Concentration of activation products in SUS304 stainless steel

\begin{tabular}{l|l}
\hline Nuclide & Concentration of activation products $(\mathrm{g} / \mathrm{t})$ \\
\hline $\mathrm{Ni}-59$ & $4.8 \mathrm{E}+01$ \\
\hline $\mathrm{Ni}-63$ & $7.8 \mathrm{E}+00$ \\
\hline Co-60 & $1.7 \mathrm{E}+00$ \\
\hline Fe-55 & $7.4 \mathrm{E}-01$ \\
\hline $\mathrm{C}-14$ & $1.7 \mathrm{E}-01$ \\
\hline Nb-36 -94 & $5.4 \mathrm{E}-02$ \\
\hline Tc-99 & $3.4 \mathrm{E}-02$ \\
\hline Mo-93 & $1.2 \mathrm{E}-02$ \\
\hline $\mathrm{K}-40$ & $1.1 \mathrm{E}-02$ \\
\hline Mn-54 & $1.8 \mathrm{E}-04$ \\
\hline Zr-93 & $1.0 \mathrm{E}-04$ \\
\hline
\end{tabular}

Except for the initial composition, the analysis conditions described in Sect. 20.3.1 were assumed. The composition data reported by the Atomic Energy Society of Japan [6] were applied in this analysis. In this reference, the concentration distributions of some elements with their mean values and standard deviations have been determined based on several measured data. The initial composition based on measured data is shown in Table 20.9 together with that based on the standard specification.

The concentration of activation products using the initial composition based on measured data is shown in Table 20.10. As a matter of course, the concentrations were changed from those in Table $20.6 \mathrm{~b}$ because the different initial compositions were assumed. It was found that Nb-94, Tc-99, Mo-93, K-40, and Zr-93 appeared in Table 20.10 because of the presence of niobium, molybdenum, and potassium in the initial composition. For the comparison with the sensitivity coefficients shown in Sect. 20.3.3, sensitivity analyses of cross sections were conducted for the several nuclides Ni-59, Ni-63, Fe-55, Co-60, Mn-54, C-14, and Cl-36, which were listed in both Tables 20.6b and 20.10.

The sensitivity coefficients of cross sections using the initial composition based on measured data are shown in Table 20.11. It was found that the results of Co-60, C-14, and Cl-36 are much different from those in Table 20.8b, which indicates that the dominant generation pathways of these nuclides were changed. Figure 20.3 shows the comparison of dominant generation pathways of Co-60, C-14, and Cl-36 between different analysis conditions. The source nuclides of Co-60, C-14, and Cl-36 were Co-59, N-14, and Cl-35, respectively, under the conditions based on measurement data, whereas those were Ni-60, C-13, and S-34, respectively, under the conditions based on the standard specification.

As shown in the foregoing example, the dominant generation pathway can be changed corresponding to the initial composition. The reliable measured data of initial impurity elements should be used if they are available. For any condition, sensitivity analyses on the basis of the methodology stated in this study can systematically identify the dominant generation pathways of activation products. 
Table 20.11 Sensitivity coefficient of cross section in SUS304 stainless steel

\begin{tabular}{l|l|l|l|l|l|l}
\hline \multirow{2}{*}{ Target nuclide } & \multicolumn{6}{l}{ Sensitivity coefficient of cross section } \\
\cline { 2 - 8 } & First largest & \multicolumn{2}{l|}{ Second largest } & \\
\hline Ni-59 & Ni-58 & $(n, \gamma)$ & 1.00 & & & \\
\hline Ni-63 & Ni-62 & $(n, \gamma)$ & 1.00 & & & \\
\hline Fe-55 & Fe-54 & $(n, \gamma)$ & 0.99 & Ni-58 & $(n, \alpha)$ & 0.01 \\
\hline Co-60 & Co-59 & $(n, \gamma)$ & 0.46 & Co-59 & $(n)_{m}$ & 0.46 \\
\hline Mn-54 & Fe-54 & $(n, \mathrm{p})$ & 1.00 & & & \\
\hline C-14 & N-14 & $(n, p)$ & 1.00 & & & \\
\hline Cl-36 & Cl-35 & $(n, \gamma)$ & 1.00 & & & \\
\hline
\end{tabular}

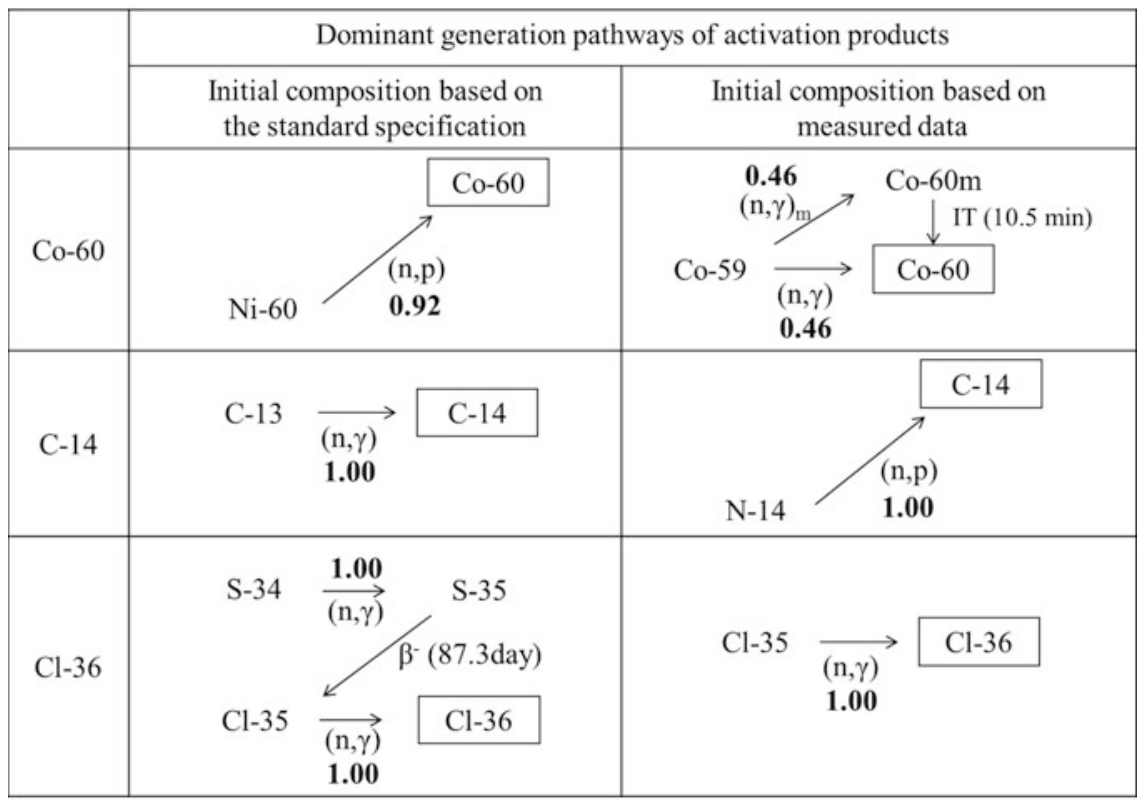

Fig. 20.3 Dominant generation pathways of activation products in SUS304 stainless steel

\subsection{Conclusion}

This study shows the sensitivity analyses of initial compositions and cross sections for activation products of in-core structure materials. The results clarified the source elements and nuclear reactions dominating the generation pathways of the activation products even for the nuclides generated through complicated pathways. The sensitivity coefficients of initial compositions are beneficial for the evaluation of the error propagated from the uncertainty of the initial composition of target materials. The sensitivity coefficients of cross sections are effective in selecting 
the objectives of nuclear reactions for the improvement of nuclear data. These results will contribute to improvement of the accuracy of numerical evaluations for the concentration of activation products.

The methodology of sensitivity analyses stated in this study is efficient for acquiring information about important impurity elements and nuclear reactions to evaluate the activation product concentrations. This methodology can be applied to the activations of ex-core structure materials if the appropriate one-group cross sections are prepared with a corresponding neutron spectrum.

Open Access This chapter is distributed under the terms of the Creative Commons Attribution Noncommercial License, which permits any noncommercial use, distribution, and reproduction in any medium, provided the original author(s) and source are credited.

\section{References}

1. Ludwig SB, Croff AG (1998) Revision to ORIGEN2: version 2.2. Transmittal memo of CCC-0371/17. Oak Ridge National Laboratory, Oak Ridge

2. Okumura K, Sugino K, Kojima K, Jin T, Okamoto T, Katakura J (2012) A set of ORIGEN2 cross section libraries based on JENDL-4.0: ORLIBJ40. JAEA Data/Code 2012-032. Japan Atomic Energy Agency [in Japanese], Ibaraki

3. Shibata K, Iwamoto O, Nakagawa T, Iwamoto N, Ichihara A, Kunieda S, Chiba S, Furukawa K, Otuka N, Ohsawa T, Murata T, Matsunobu H, Zukaran A, Kameda S, Katakura J (2011) JENDL-4.0: a new library for nuclear science and engineering. J Nucl Sci Technol 48(1):1-30

4. Namekawa M, Fukahori T (2012) Tables of nuclear data (JENDL/TND-2012). JAEA Data/ Code 2012-014. Japan Atomic Energy Agency, Ibaraki

5. Evans JC, Lepel EL, Sanders RW, Wilkerson CL, Silker W, Thomas CW, Abel KH, Robertson DR (1984) Long-lived activation products in reactor materials. NUREG/CR-3474. U.S. Nuclear Regulatory Commission, Washington

6. Atomic Energy Society of Japan (2010) AESJ-SC-F015: basic procedure to determine the activity concentration of sub-surface disposal waste [in Japanese] 